\title{
Über das Auftreten eines Tripelsalzes aus wässerigen Lösungen ohne gleichzeitiger Bildung eines binären Doppel- salzes.
}

\author{
Von \\ Robert Kremann und Hans Rodemund. ${ }^{1}$ \\ Mit 2 Figuren im Text.
}

Gelegentlich von Versuchen betreffend die Umsetzung des als Nebenprodukt des Le Buancschen Sodaprozesses erhaltenen Calciumthiosulfats, haben wir auch das reziproke Salzpaar

$$
\mathrm{CaS}_{2} \mathrm{O}_{3}+2 \mathrm{NaNO}_{3} \rightleftarrows \mathrm{Na}_{2} \mathrm{~S}_{2} \mathrm{O}_{3}+\mathrm{Ca}\left(\mathrm{NO}_{3}\right)_{2}
$$

bei 9 und $25^{0}$ untersucht.

Über die gesamten Untersuchungen haben wir an anderer Stelle bereits berichtet und die gegenseitigen Löslichkeitsverhältnisse obigen Salzpaares nach der Meyerhoffer-VAn'T Hoffschen Methode zur graphischen Darstellung gebracht.

Es war festgestellt worden, daB auBer den vier Salzen bei beiden 'Temperaturen, und zwar als

$$
\mathrm{NaNO}_{3}, \mathrm{Ca}\left(\mathrm{NO}_{3}\right)_{2} .4 \mathrm{aq}, \mathrm{Ca}_{2} \mathrm{~S}_{2} \mathrm{O}_{3} .6 \text { aq und } \mathrm{Na}_{2} \mathrm{~S}_{2} \mathrm{O}_{3} .5 \mathrm{aq}
$$

keine binären Doppelsalze auftreten. Wohl aber grenzt sich im Gebiet der ternären Salzlösungen ein Existenzfeld eines ternären Doppelsalzes $\mathrm{NaNO}_{3} \cdot \mathrm{CaS}_{2} \mathrm{O}_{3} \cdot \mathrm{Na}_{2} \mathrm{~S}_{2} \mathrm{O}_{3} .11 \mathrm{aq}$ ab, das mit steigender Temperatur kleiner wird und nur eine derartige Verschiebung erfährt, daB es bei bestimmter Temperatur in die Einzelsalze zerfälit. Dieser nonvariante Umwandlungspunkt ergab sich aus Löslichkeitsverhältnissen zu $29^{\circ}$, auf thermischem Wege zu $29.2^{\circ}$.

Es sei uns nun gestattet, die Löslichkeitsverhältnisse obigen reziproken Salzpaares an dieser Stelle nach der schöneren und übersichtiicheren JäNNECKEschen Methode der Darstellung wiederzugeben.

1 Sitzungsber, der Wiener Akad. 1914, Sitzung v. 5. März 1914.

Z. anorg. Chem. Bd. 86 . 
JäNNECKE drückt bekanntlich die Löslichkeit reziproker Salzpaare statt in Gramm oder Molen Salz in einer besonderen Menge Lösung oder Wasser in folgender Weise aus. Es werden also die Mole Wasser angegeben, die notwendig sind, um je $100 \mathrm{Mol}$ reinen Salzes oder je $100 \mathrm{Mol}$ zweier oder dreier Salze zu lösen. Man kann dann für eine bestimmte Temperatur das Verhältnis der vier Salze der reziproken Salzpaare in Lösung durch ein Quadrat, dessen Seiten 100 Mol entsprechen, veranschaulichen. Man trägt hierbei nicht die Löslichkeit der Salze als solche auf, sondern die der Ionen, wobei im betrachteten Falle, der Äquivalenz halber, die Natriumionen als $\mathrm{Na}_{2} \cdot$ und die Nitrationen als $\left(\mathrm{NO}_{3}{ }^{*}\right)_{2}$ zur Auftragung kommen. Ist allgemein der Gehalt an Calciumionen $a$, an Thiosulfationen $b$, so tragen wir auf der Quadratseite $D C$ von $D$ aus (Fig. 1 u. 2) den Wert $a$, auf der Quadratseite $A D$ den Wert $b$ von $A$ aus auf. Dann sind, da wir ja stets 100 Mol Salz oder Salzgemisch betrachten, infolge der Äquivalenz der positiven und negativen Ionen die Gehalte an $\mathrm{Na}_{2}{ }^{*}$ implizite durch $100-a$, an $\left(\mathrm{NO}_{3}{ }^{*}\right)_{2}$ durch $100-b$ gegeben. In den Quadraten entsprechen die Eckpunkte:

$$
\begin{aligned}
& A \text { einer reinen Lösung von } \mathrm{NaNO}_{3} \\
& B \quad " \quad, \quad, \quad, \quad \mathrm{Ca}\left(\mathrm{NO}_{3}\right)_{2} \\
& C \quad " \quad " \quad " \quad \text { " } \quad \mathrm{Ca}_{2} \mathrm{~S}_{2} \mathrm{O}_{3} \\
& D \quad " \quad, \quad, \quad, \quad \mathrm{Na}_{2} \mathrm{~S}_{2} \mathrm{O}_{3}
\end{aligned}
$$

In den Quadratseiten liegen die Lösungen, die je zwei Salze enthalten.

$$
\begin{aligned}
& A B \text {. . . } \mathrm{CaNO}_{3} \text { und } \mathrm{NaNO}_{3} \\
& B C \text {. . . } \mathrm{CaNO}_{3} \quad, \mathrm{Ca}_{2} \mathrm{~S}_{2} \mathrm{O}_{3} \\
& C D \text {. . . } \mathrm{Na}_{2} \mathrm{~S}_{2} \mathrm{O}_{3}, \mathrm{Ca}_{2} \mathrm{~S}_{2} \mathrm{O}_{3} \\
& D A \text {. . . } \mathrm{Na}_{2} \mathrm{~S}_{2} \mathrm{O}_{3}, \quad \mathrm{NaNO}_{3}
\end{aligned}
$$

während im Innern des Quadrates sämtliche Lösungen mit drei Salzen zu liegen kommen. Die zugehörige Zahl $M$, die Mol Wasser, die nötig sind, um je $100 \mathrm{Mol}$ Salz oder Salzgemisch zu lösen, werden den entsprechenden Punkten beigeschrieben. Die folgenden beiden Tabellen geben die einzelnen Fixpunkte bei 9 und bei $25^{\circ}$, wie sie zur Abgrenzung der Existenzfelder der einzelnen Bodenkörper von Bedeutung sind, in der auseinandergesetzten Berechnungsart wieder. In Fig. 1 sind die Löslichkeitsverhältnisse des reziproken Salzpares bei $9^{\circ}$, in Fig. 2 bei $25^{\circ}$ nach der JüNNECKEschen Metbode zur Darstellung gebracht. 
Über das Auftreten eines Tripelsalzes aus wässerigen Lösungen usw. $37 \check{5}$

\begin{tabular}{|c|c|c|c|c|c|c|c|c|c|c|c|c|c|c|c|}
\hline & $\begin{array}{l}O^{\pi} \mathrm{S} \\
=q^{2}\end{array}$ & $\|=088$ & o & 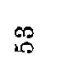 & $=$ & $\stackrel{8}{8}$ & $\pi$ & $\infty$ & 药 & $=$ & $\mathbb{q}$ & के & $\stackrel{10}{0}$ & 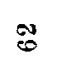 & $\Leftrightarrow$ \\
\hline & $\begin{array}{l}.8 \times \\
=0 \\
=0\end{array}$ & $0 \%$ & \& & 0 & $\S$ & 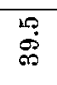 & $\bar{\infty}$ & $\mathscr{8}$ & $\underset{\oplus}{\stackrel{0}{H}}$ & 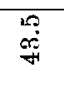 & $\overrightarrow{0}$ & 要 & 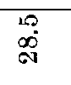 & 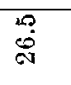 & 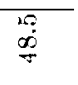 \\
\hline & 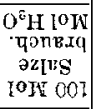 & 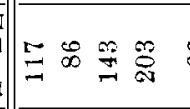 & 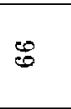 & $\stackrel{\ominus}{\varrho}$ & $\ddot{\infty}$ & $\underset{\sim}{\stackrel{H}{*}}$ & 8 & $\stackrel{\circ}{\circ}$ & $\mathscr{\infty}$ & ్ㅗㅁ & $\vec{\infty}$ & ஓ & 28 & $\mathscr{g}$ & $\vec{\infty}$ \\
\hline & 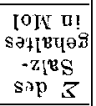 & 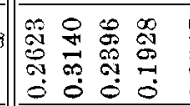 & 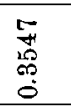 & 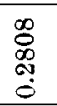 & 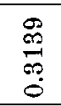 & 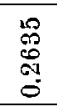 & 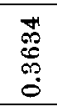 & 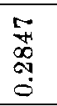 & \% & 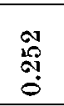 & 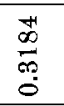 & $\begin{array}{l}\overrightarrow{0} \\
0 \\
0 \\
0 \\
0 \\
0\end{array}$ & 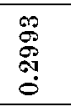 & $\begin{array}{l}0 \\
0 \\
0 \\
0 \\
0 \\
0\end{array}$ & $\underset{\substack{\infty \\
e}}{\stackrel{\infty}{c}}$ \\
\hline & $\begin{array}{l}0^{z} \mathrm{H} \\
\mathrm{l}^{0} \mathrm{~N}\end{array}$ & 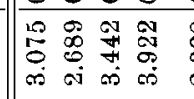 & 离 & 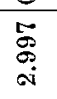 & 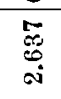 & 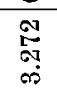 & 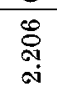 & $\underset{\infty}{\stackrel{\infty}{\oplus}}$ & 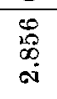 & 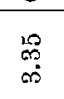 & 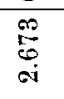 & $\begin{array}{l}8 \\
8 \\
0 \\
0\end{array}$ & 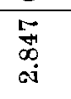 & 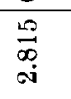 & 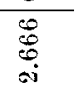 \\
\hline & 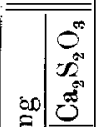 & 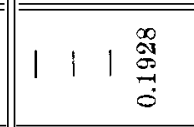 & 1 & 1 & $\begin{array}{l}10 \\
0 \\
0 \\
0 \\
0\end{array}$ & $\frac{7}{0}$ & $\stackrel{9}{8}$ & $\begin{array}{l}\stackrel{r}{D} \\
\stackrel{0}{0} \\
\end{array}$ & $\begin{array}{l}\text { 量 } \\
\text { 要 } \\
0 \\
0\end{array}$ & $\stackrel{0}{\vdots}$ & 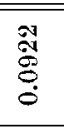 & $\underset{\stackrel{\infty}{0}}{\stackrel{0}{0}}$ & $\begin{array}{l}5 \\
10 \\
0 \\
0 \\
0\end{array}$ & $\stackrel{5}{\circ}$ & $\frac{5}{0}$ \\
\hline č & 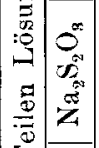 & 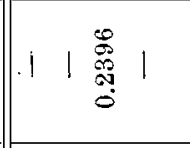 & 1 & 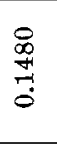 & 1 & 莒 & 1 & 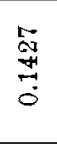 & $\underset{0}{\stackrel{N}{*}}$ & 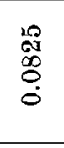 & 1 & 1 & $\begin{array}{l}0 \\
0 \\
0 \\
0 \\
0 \\
0\end{array}$ & 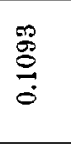 & 1 \\
\hline & 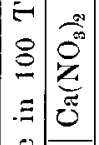 & $|\underset{\substack{0 \\
0}}{0}| 1 \mid$ & 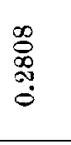 & 1 & 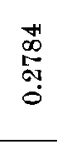 & 1 & $\begin{array}{l}\vec{\infty} \\
\stackrel{\infty}{\sharp} \\
\stackrel{4}{0}\end{array}$ & 1 & 1 & & $\underset{0}{\stackrel{5}{0}}$ & $\begin{array}{l}9 \\
9 \\
0 \\
0 \\
0\end{array}$ & 1 & 1 & $\begin{array}{l}\stackrel{9}{0} \\
\stackrel{9}{:} \\
\stackrel{0}{0}\end{array}$ \\
\hline 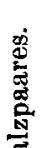 & 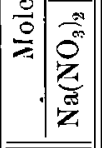 & $\|$ & $\begin{array}{l}\stackrel{9}{8} \\
\stackrel{8}{0} \\
\stackrel{0}{0}\end{array}$ & 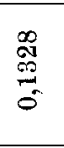 & 1 & 1 & 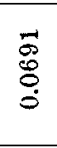 & 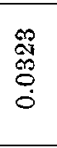 & 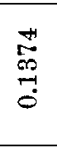 & $\stackrel{8}{8}$ & $\stackrel{20}{\stackrel{20}{0}}$ & $\begin{array}{l}\text { 渵 } \\
: \\
0 \\
0\end{array}$ & $\begin{array}{l}8 \\
\stackrel{0}{0} \\
0\end{array}$ & 总 & $\begin{array}{l}\overrightarrow{0} \\
\stackrel{0}{0}\end{array}$ \\
\hline & 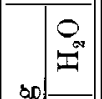 & 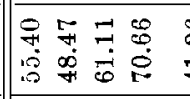 & 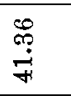 & $\begin{array}{l}\overrightarrow{0} . \\
\text { ti } \\
\text { in }\end{array}$ & $\begin{array}{l}\overrightarrow{5} \\
\overrightarrow{5} \\
\overrightarrow{+} \\
\vec{T}\end{array}$ & 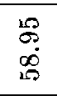 & 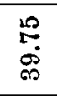 & & & $\ddot{8}$ & t & 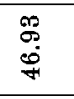 & 疍. & 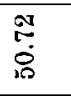 & $\begin{array}{l}\infty \\
0 \\
\infty \\
0 \\
+\end{array}$ \\
\hline & 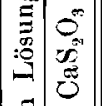 & 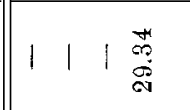 & 1 & 1 & $\begin{array}{c}\overrightarrow{0} \\
0 \\
0\end{array}$ & 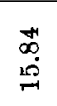 & $\stackrel{F}{F}$ & $=$ & 0 & $\stackrel{\infty}{\stackrel{\infty}{0}}$ & $\begin{array}{l}\stackrel{H}{0} \\
\stackrel{+}{ \pm}\end{array}$ & $\begin{array}{l}18 \\
9 \\
10\end{array}$ & 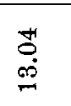 & 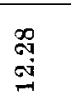 & 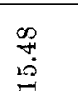 \\
\hline & 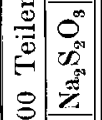 & 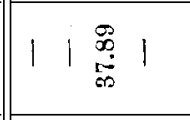 & I & 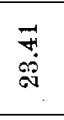 & 1 & 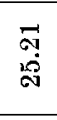 & 1 & 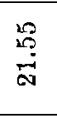 & $\stackrel{\infty}{\rightarrow}$ & 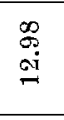 & 1 & $!$ & $\begin{array}{l}\infty \\
\stackrel{\infty}{+} \\
\infty \\
\infty\end{array}$ & 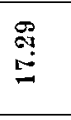 & 1 \\
\hline & 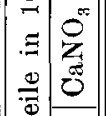 & 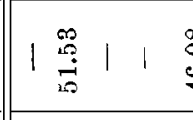 & $\begin{array}{l}\infty \\
\stackrel{+}{+} \\
+\end{array}$ & 1 & $\begin{array}{l}\infty \\
\vdots \\
0 \\
\dot{+}+1\end{array}$ & 1 & $\begin{array}{c}\stackrel{N}{N} \\
\stackrel{H}{\sigma}\end{array}$ & 1 & 1 & 1 & 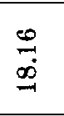 & $\underset{+}{\stackrel{9}{+1}}$ & 1 & 1 & 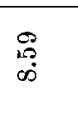 \\
\hline & 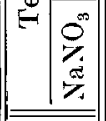 & $\mid$\begin{tabular}{lllll}
0 & 0 \\
\hdashline & 1 & 1 & 1 & 9 \\
& & & &
\end{tabular} & 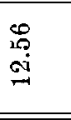 & 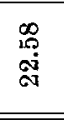 & 1 & 1 & $\stackrel{\substack{\stackrel{\infty}{\leftrightarrows} \\
=}}{=}$ & 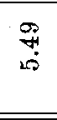 & 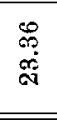 & $\stackrel{0}{\circ}$ & $\begin{array}{l}\stackrel{\#}{0} \\
\stackrel{\sim}{\rightleftarrows}\end{array}$ & $\begin{array}{l}\stackrel{9}{0} \\
\stackrel{0}{0} \\
\stackrel{0}{0}\end{array}$ & 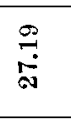 & 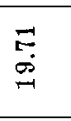 & $\underset{8}{\stackrel{8}{8}}$ \\
\hline & 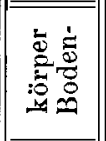 & \multicolumn{14}{|c|}{ 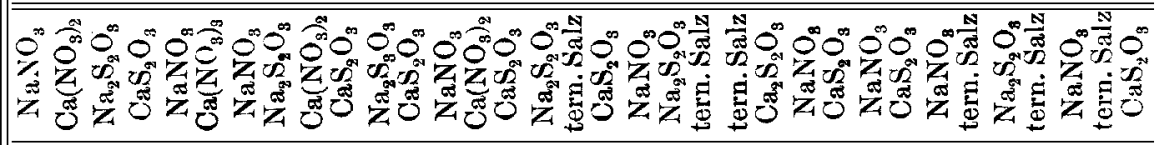 } \\
\hline & 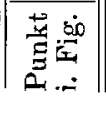 & $\| \mp$ & $\theta$ & $E$ & 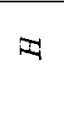 & 0 & 2 & 25 & 29 & $s^{5}$ & $22^{\infty}$ & $a^{\infty}$ & $\begin{array}{c}2 \underbrace{\infty} \\
26^{\infty} \\
2 *\end{array}$ & RE & RE \\
\hline
\end{tabular}




\begin{tabular}{|c|c|c|c|c|c|c|c|c|c|c|c|c|c|c|c|c|c|}
\hline & 0 & $\|^{0} \circ 8$ & 0 & 8 & 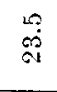 & $\S$ & 8 & $\stackrel{\infty}{\sim}$ & 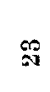 & $\infty$ & बi & 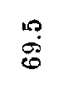 & 电 & $\ddot{\infty}$ & 운 & $\infty$ & $\frac{10}{3}$ \\
\hline & $\tilde{\sigma}$ & $10 \underset{0}{8}$ & 䑰 & 0 & 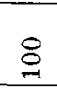 & $\S$ & $\frac{19}{9}$ & 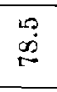 & $\underset{\dot{D}}{\stackrel{8}{8}}$ & $\begin{array}{l}10 \\
\dot{8} \\
\end{array}$ & 吕 & $\stackrel{\infty}{\infty}$ & 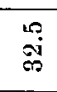 & $\stackrel{10}{\stackrel{9}{+1}}$ & 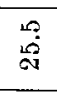 & P & $\stackrel{\ominus}{\dot{b}}$ \\
\hline & 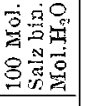 & 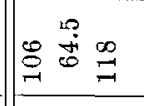 & $\stackrel{9}{+1}$ & $\stackrel{\substack{0 \\
\infty}}{\infty}$ & $\stackrel{\infty}{\stackrel{\infty}{0}}$ & $\stackrel{8}{2}$ & $\stackrel{5}{+\infty}$ & 穼 & 量 & $\begin{array}{l}\text { a } \\
\text { 唡 }\end{array}$ & $\stackrel{10}{10}$ & $\begin{array}{l}0 \\
i 8 \\
18\end{array}$ & $\stackrel{\circ}{+}$ & $\begin{array}{l}100 \\
00 \\
0\end{array}$ & $\stackrel{\mathfrak{r}}{\mathbf{r}}$ & 象 & $\stackrel{P}{i}$ \\
\hline & 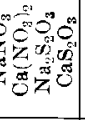 & 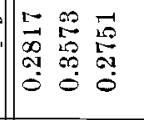 & 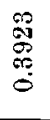 & 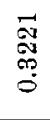 & $\begin{array}{l}\infty \\
0 \\
0 \\
0 \\
0 \\
0\end{array}$ & 我 & 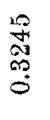 & 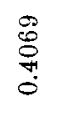 & $\begin{array}{l}\infty \\
\infty \\
\infty \\
\infty \\
0 \\
0\end{array}$ & $\begin{array}{l}\stackrel{0}{0} \\
0 \\
0 \\
0 \\
0\end{array}$ & $\begin{array}{l}0 \\
0 \\
0 \\
0 \\
0 \\
0 \\
0\end{array}$ & 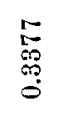 & 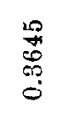 & 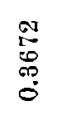 & $\begin{array}{l}\stackrel{5}{\infty} \\
\stackrel{0}{m} \\
0\end{array}$ & 点 & $\begin{array}{l}\infty \\
0 \\
\stackrel{0}{0} \\
0 \\
0\end{array}$ \\
\hline & $\overline{\bar{\sigma}}$ & 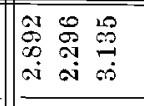 & 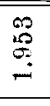 & 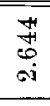 & 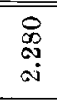 & 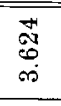 & 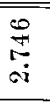 & 我 & 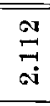 & $\begin{array}{l}0 \\
0 \\
0 \\
0 \\
01 \\
\end{array}$ & $\begin{array}{l}\vec{b} \\
\vec{a} \\
\mathbf{a}\end{array}$ & 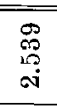 & 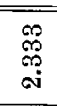 & $\underset{\text { స̆ }}{\vec{i}}$ & $\underset{\substack{\infty \\
\infty}}{\infty}$ & 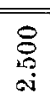 & $\underset{B}{\stackrel{B}{H}}$ \\
\hline & 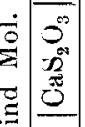 & 111 & 1 & 1 & $\begin{array}{l}\text { it } \\
i \infty \\
0 \\
0 \\
0 \\
\end{array}$ & 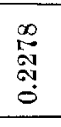 & $\begin{array}{c}\stackrel{0}{\circ} \\
\stackrel{9}{9} \\
\\
0\end{array}$ & 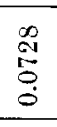 & $\begin{array}{l}-1 \\
0 \\
0 \\
0 \\
0 \\
\end{array}$ & $\begin{array}{l}5 \\
0 \\
0 \\
0\end{array}$ & $\begin{array}{l}4 \\
1 \\
0 \\
0 \\
0 \\
0\end{array}$ & $\begin{array}{l}0 \\
\stackrel{1}{0} \\
0 \\
0 \\
\end{array}$ & 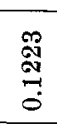 & $\stackrel{5}{\frac{\pi}{3}}$ & 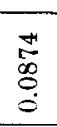 & $\begin{array}{l}\overrightarrow{5} \\
\stackrel{0}{0} \\
0\end{array}$ & 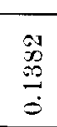 \\
\hline & $\mid$ & $11 \underset{\substack{5 \\
\hdashline}}{\stackrel{5}{5}}$ & 1 & 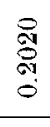 & I & 1 & $\frac{\mathscr{8}}{\stackrel{0}{0}}$ & 1 & 1 & 1 & 1 & 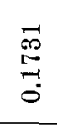 & 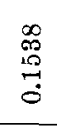 & $\begin{array}{l}\infty \\
\substack{\infty \\
0 \\
0 \\
0} \\
\end{array}$ & $\begin{array}{l}\overrightarrow{6} \\
\stackrel{6}{6} \\
0\end{array}$ & $\begin{array}{l}-5 \\
0 \\
0 \\
0\end{array}$ & $\frac{18}{5}$ \\
\hline & 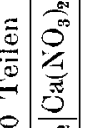 & 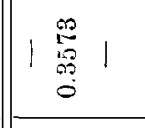 & 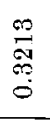 & 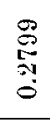 & 1 & I & 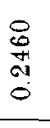 & 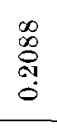 & 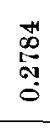 & 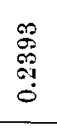 & 1 & 1 & I & 1 & 1 & 1 & 1 \\
\hline & 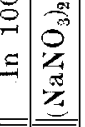 & 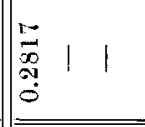 & $\stackrel{0}{\stackrel{5}{0}}$ & $\begin{array}{l}\vec{\Xi} \\
\stackrel{心}{0} \\
0\end{array}$ & 1 & 1 & 1 & $\begin{array}{l}1 \\
0 \\
0 \\
0 \\
0 \\
0\end{array}$ & $\begin{array}{l}\infty \\
\infty \\
0 \\
0 \\
0\end{array}$ & $\begin{array}{l}\stackrel{9}{+} \\
\stackrel{+}{0} \\
0 \\
\end{array}$ & $\stackrel{\infty}{\frac{\infty}{10}}$ & $\begin{array}{l}0 \\
\stackrel{0}{0} \\
\stackrel{0}{0} \\
0\end{array}$ & $\begin{array}{l}\vec{D} \\
\infty \\
0 \\
0 \\
0\end{array}$ & $\begin{array}{l}01 \\
0 \\
0 \\
0 \\
0\end{array}$ & $\begin{array}{l}\stackrel{0}{0} \\
\stackrel{8}{8} \\
\stackrel{8}{8}\end{array}$ & $\stackrel{20}{\vec{F}}$ & $\begin{array}{l}\overrightarrow{0} \\
\stackrel{9}{9} \\
0\end{array}$ \\
\hline & $=$ & 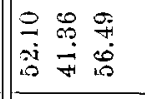 & 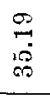 & 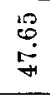 & 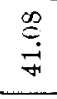 & 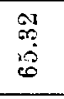 & 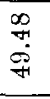 & 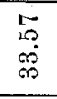 & 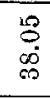 & $\begin{array}{l}\overline{0} \\
\dot{0} \\
\end{array}$ & 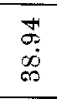 & 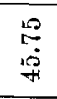 & 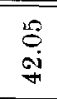 & $\underset{\mathrm{I}}{\mathrm{S}}$ & 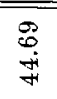 & 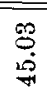 & 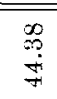 \\
\hline & 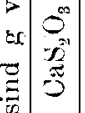 & 111 & 1 & 1 & $\stackrel{8}{8}$ & $\begin{array}{l}\infty \\
\stackrel{\infty}{0} \\
\stackrel{\infty}{0}\end{array}$ & 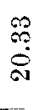 & $\stackrel{\mathscr{g}}{\stackrel{\leftrightarrow}{=}}$ & $\stackrel{\oplus}{\stackrel{m}{m}}$ & $\begin{array}{l}\infty \\
\infty \\
+i \\
+i\end{array}$ & \begin{tabular}{l}
8 \\
\hdashline \\
01
\end{tabular} & $\stackrel{\infty}{\leftrightarrow}$ & $\begin{array}{l}\vec{b} \\
\dot{D} \\
\end{array}$ & 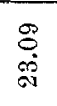 & 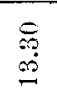 & $\stackrel{\infty}{=}$ & $\stackrel{3}{3}$ \\
\hline & 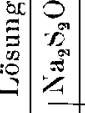 & $1 \quad 1 \vec{b}$ & 1 & $\stackrel{s}{\stackrel{s}{\infty}}$ & I & 1 & $\stackrel{\infty}{\stackrel{\infty}{\circ}}$ & 1 & I & 1 & 1 & $\begin{array}{l}\infty \\
\substack{\infty \\
\infty \\
\infty \\
\infty}\end{array}$ & 草 & 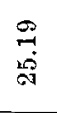 & $\stackrel{R}{R}$ & ț & $\stackrel{9}{\stackrel{9}{ت}}$ \\
\hline
\end{tabular}

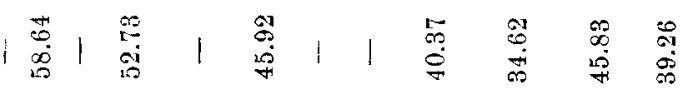

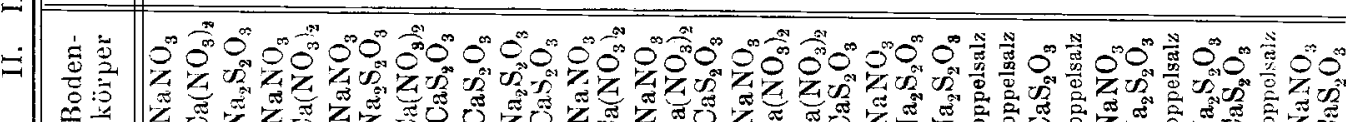

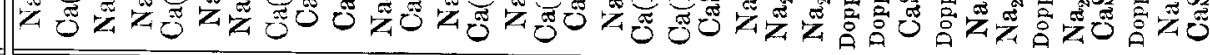

Imir 
Die Punktbezeichnung ist in beiden Figuren eine ganz analoge. Es haben die Punkte der Reihe nach folgende Bedeutung:

$A$ entspr. Sättigung an . . . $\mathrm{NaNO}_{3}$

$B \quad, \quad, \quad, \quad$. . . $\mathrm{Ca}\left(\mathrm{NO}_{3}\right)_{2} .4 \mathrm{aq}$

$C \quad " \quad$ " $"$. . . $\mathrm{CaS}_{2} \mathrm{O}_{3} .6 \mathrm{aq}$

D $", \quad " \quad$. . . $\mathrm{Na}_{3} \mathrm{~S}_{2} \mathrm{O}_{3} .5 \mathrm{aq}$

$E \quad, \quad$ gleichzeit. Sättigung an $\mathrm{CaNO}_{3} .4 \mathrm{aq}$ u. $\mathrm{NaNO}_{3}$

$F \quad, \quad, \quad, \quad \mathrm{NaNO}_{3} .5 \mathrm{aq}$ u. $\mathrm{Na}_{2} \mathrm{~S}_{2} \mathrm{O}_{3} .5 \mathrm{aq}$

G " " " " $\quad \mathrm{Na}_{2} \mathrm{~S}_{2} \mathrm{O}_{3} \cdot 5 \mathrm{aq}$ u. $\mathrm{CaS}_{2} \mathrm{O}_{3} \cdot 6 \mathrm{aq}$

$H \quad$ " " " " $\mathrm{CaS}_{2} \mathrm{O}_{3} \cdot 6 \mathrm{aq}$ u. $\mathrm{Ca}\left(\mathrm{NO}_{3}\right)_{2} \cdot 4 \mathrm{aq}$

$R_{1} \quad " \quad$ " " $\quad \mathrm{CaS}_{2} \mathrm{O}_{3} \cdot 6 \mathrm{aq}, \mathrm{Na}_{2} \mathrm{~S}_{2} \mathrm{O}_{3}$ u. Tripelsalz

$R_{2} \quad " \quad " \quad$ " $\mathrm{Na}_{2} \mathrm{~S}_{2} \mathrm{O}_{3} \cdot 5 \mathrm{aq}, \mathrm{NaNO}_{3}$ u. ",

$R_{3} \quad, \quad$ " " " $\mathrm{NaNO}_{3} \mathrm{CaS}_{2} \mathrm{O}_{3} \cdot 6 \mathrm{aq}$ u. ",

$P \quad$ " " " " $\mathrm{CaNO}_{3} .4 \mathrm{aq} \mathrm{NaNO}_{3}$ u. $\mathrm{CaS}_{2} \mathrm{O}_{3} \cdot 6$ all

Die Kurven in den beiden Figuren haben die folgende Bedeutung:

HP gleichzeitige Sättigung an . . $\mathrm{CaS}_{2} \mathrm{O}_{3} .6 \mathrm{aqu}$ u. $\mathrm{Ca}\left(\mathrm{NO}_{3}\right)_{2} \cdot 4 \mathrm{aq}$ $E P$

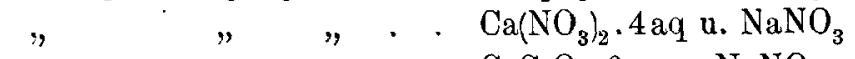

$P R_{3} \quad$ " $\quad$ " $\quad$. . $\mathrm{CaS}_{2} \mathrm{O}_{3} \cdot 6$ aq u. $\mathrm{NaNO}_{3}$

$R_{3} R_{1} \quad " \quad \quad$ " $\quad$. . . $\mathrm{CaS}_{2} \mathrm{O}_{3} .6$ aq u. Tripelsalz

$R_{3} R_{2} \quad$ "

$\mathrm{NaNO}_{3}$ u. .,

$R_{2} R_{1} \quad$ " " " $\quad$ ". . $\mathrm{Na}_{2} \mathrm{~S}_{2} \mathrm{O}_{3} 5$.aqu. ,"

$R_{2} F \quad " \quad, \quad \# \quad . \quad . \quad \mathrm{NaNO}_{3}$ u. $\mathrm{Na}_{2} \mathrm{~S}_{2} \mathrm{O}_{3} \cdot 5 \mathrm{aq}$

$R_{1} G \quad " \quad " \quad, \quad . \quad \mathrm{NaS}_{2} \mathrm{O}_{3} .5 \mathrm{aq}$ u. $\mathrm{CaS}_{2} \mathrm{O}_{3} \cdot 6 \mathrm{a}$

Die durch die Kurven abgegrenzten Existenzfelder der einzelnen Salze sind die folgenden:

$B H P E$ entspricht dem Existenzfeld von $\mathrm{Ca}\left(\mathrm{NO}_{3}\right)_{2} .4 \mathrm{aq}$

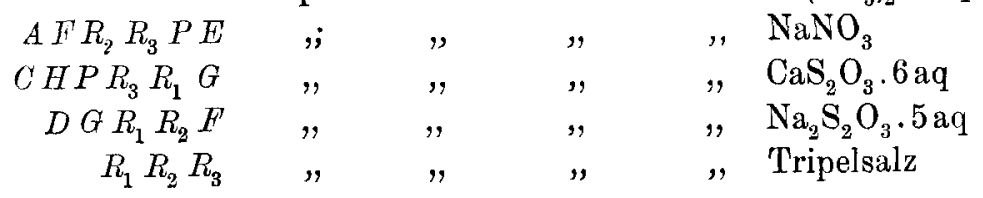

Aus dem Vergleich der Existenzfelder von Tripelsalz bei 9 und $25^{\circ}$ sieht man deutlich, daß mit steigender Temperatur das Existenzfeld des Tripelsalzes kleiner wird. Diese Verkleinerung erfolgt durch eine relativ geringe Verschiebung des Punktes $R_{1}$ und der relativ größeren Verschiebung des Punktes $R_{2}$ und in starkem Maße von Punkt $R_{3}$. Man sieht aus dieser Verschiebung, daB bei 
hoher Temperatur das Existenzfeld zusammenschrumpfen muB und keine derartige Verschiebung erfabren kann, daB etwa bei hoher

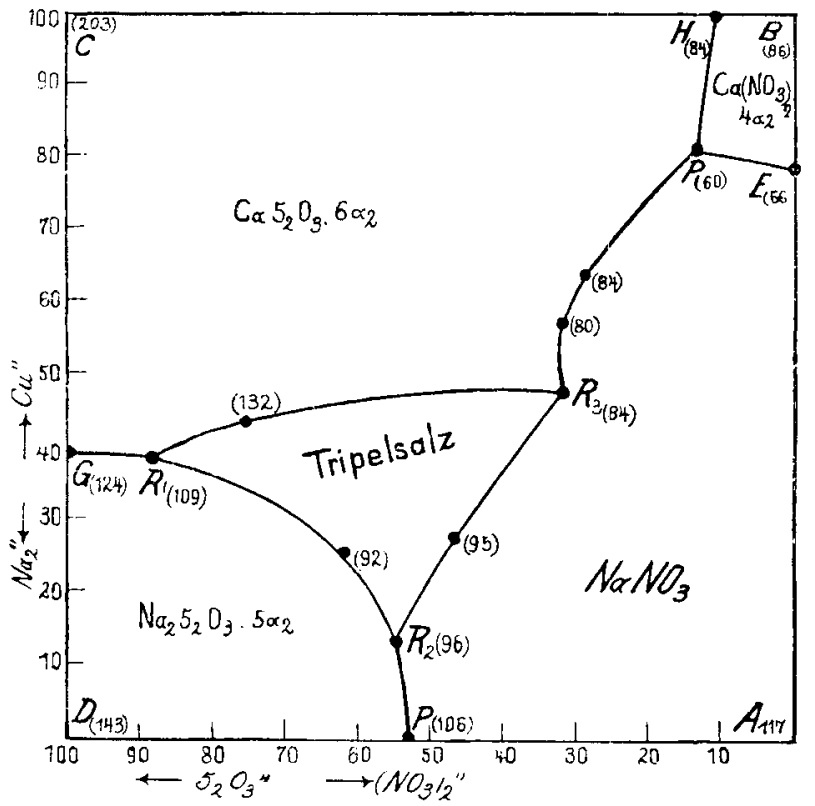

Fig. 1.

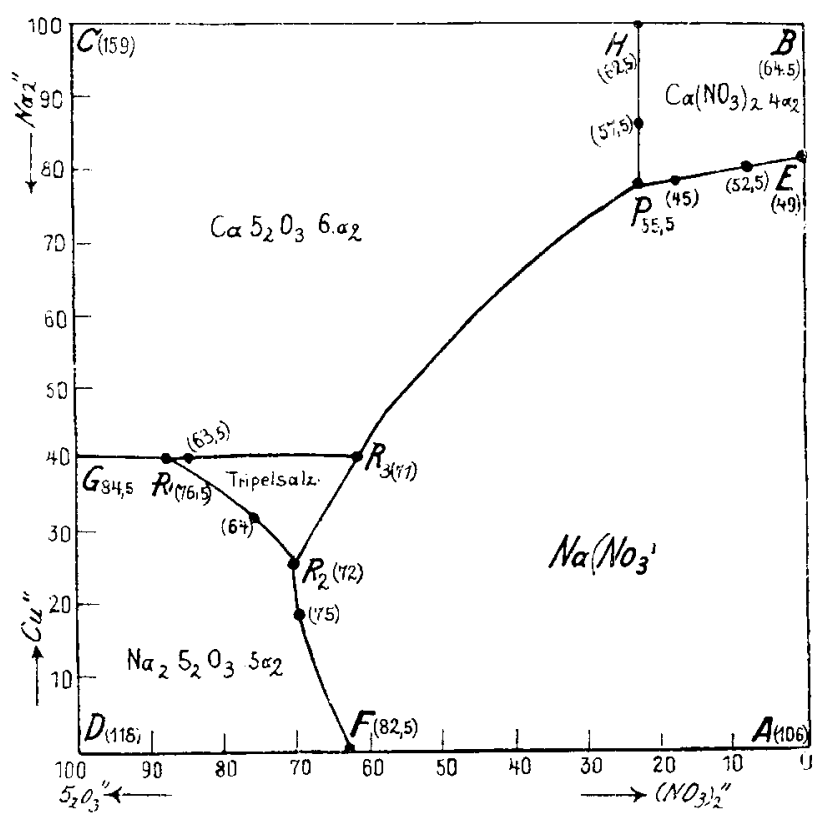

Fig. 2. 
Über das Auftreten eines Tripelsalĩes aus wö̈serigen Lüsungen usw. 379

Temperatur es zu einem Schnitt mit der Quadratseite kommen kann, d. h. es wandelt sich das Tripelsalz nicht primär in ein binäres Doppelsalz um, sondern es zerfällt direkt in die einzelnen Salze.

Wie bereits eingangs erwähnt, wurde diese Umwandlungstemperatur thermisch zu $29.2^{\circ}$ festgelegt, während die Löslichkeitsbestimmung aus reinem Doppelsalz den wenig verschiedenen Wert von ca. $29^{\circ}$ ergab.

Grax, Chemisches Laboratorium der Universität, 21. Februar 1914.

Bei der Redaktion eingegangen am 25. Februar 1914。 